\title{
L’espérance de scolarisation (ou de vie scolaire) des jeunes au Québec
}

\author{
THE EDUCATIONAL EXPECTANCY OF YOUNG PEOPLE IN \\ QUEBEC
}

\section{LA ESPERANZA DE ESCOLARIZACIÓN (O DE VIDA ESCOLAR) DE LOS JÓVENES EN QUEBEC}

\section{André Lespérance}

Volume 23, numéro 1, printemps 1994

L'enfance

URI : https://id.erudit.org/iderudit/010164ar

DOI : https://doi.org/10.7202/010164ar

\section{Aller au sommaire du numéro}

\section{Éditeur(s)}

Association des démographes du Québec

\section{ISSN}

0380-1721 (imprimé)

1705-1495 (numérique)

Découvrir la revue

\section{Citer cette note}

Lespérance, A. (1994). L'espérance de scolarisation (ou de vie scolaire) des jeunes au Québec. Cahiers québécois de démographie, 23(1), 103-120. https://doi.org/10.7202/010164ar

\section{Résumé de l'article}

De l'école primaire à l'université, la scolarisation totale dispensée en 1992-1993, correspond en moyenne à 15,3 années par Québécois et Québécoise en âge de fréquenter un établissement scolaire. Cette mesure est la somme des taux de scolarisation par année d'âge pendant une année scolaire donnée. L'espérance de scolarisation au Québec a augmenté de 0,9 année depuis 1987-1988 et se situe parmi les plus élevées au monde. Les gains récents ont été effectués en partie par du rattrapage au primaire et au secondaire réalisé au secteur des adultes. Pour le reste, les gains se situent à l'enseignement collégial ( 0,3 année) et à l'enseignement universitaire (0,3 année). L'allongement de l'espérance de scolarisation aux niveaux post-secondaires s'explique essentiellement par une accessibilité plus large.

Les Québécoises affichent dans l'ensemble une scolarisation supérieure à celle des hommes. Au primaire et au secondaire, la durée de leur scolarisation est moindre que celle des hommes parce qu'elles perdent moins de temps en redoublements. Parce que les femmes accèdent en plus grand nombre au collège et à l'université, en 1992-1993, leur espérance de scolarisation aux niveaux supérieurs est de 3,7 années, contre 2,9 années pour les hommes. 
Cahiers québécois de démographie

Vol. 23, no 1, printemps 1994, p. 103-120.

\title{
L'espérance de scolarisation lou de vie scolaire) des jeunes au Guébec
}

\author{
André LESPÉRANCE *
}

Ce texte présente une forme d'indicateur de scolarisation relativement inusitée jusqu'à ces dernières années. Il s'agit de la "durée probable de scolarisation" ou "espérance de vie scolairen, que nous appellerons plus simplement espérance de scolarisation. Le concept n'est pas nouveau en démographie puisqu'il s'agit d'une "somme d'événements réduits" appliquée au domaine scolaire. Des études du ministère de l'Éducation du Québec, réalisées depuis quelques années, ont déjà utilisé un indice analogue à des fins particulières (Lespérance, 1980). Plus récemment, la France, l'UNESCO et le Québec en ont élargi la diffusion en incluant cette mesure dans leurs publications sur les indicateurs de l'éducation.

La description du phénomène de la scolarisation est généralement abordée avec des taux par âge. Ainsi est-on habitué à considérer, pour un âge donné, la proportion des personnes qui fréquentent un établissement scolaire, soit à temps complet, soit à temps partiel; on notera par exemple que $24,6 \%$ des jeunes de 15-19 ans fréquentent un établissement collégial au Québec en 1992-1993. Cependant, si l'on veut décrire l'ensemble du phénomène de la scolarisation, ce taux doit être mis en regard des taux observés à d'autres âges ou à d'autres ordres d'enseignement ${ }^{1}$. Il faut donc disposer d'une batterie de taux de scolarisation qui devient vite trop lourde à décrire et cela incite souvent à ne porter attention qu'à certains

* Ministère de l'Éducation du Guébec, Direction des statistiques et des études quantitatives.

1 Ordre d'enseignement : "Chacune des grandes divisions de l'enseignement. [...] Au Québec, les ordres d'enseignement sont : l'enseignement primaire; l'enseignement secondaire; l'enseignement collégial; l'enseignement universitaire* (R. Legendre, Dictionnaire actuel de l'éducation, Larousse). 
âges où se concentre la scolarisation. Si, par contre, on dit que chaque jeune Québécois passe en moyenne 1,75 an à l'enseignement collégial et 15,28 ans dans l'ensemble du système scolaire, le portrait de la scolarisation sera plus complet et tout aussi précis (bien que moins détaillé) qu'avec des séries de taux puisque chacun des taux est alors mis en perspective. La mesure présentée ici est la synthèse de tous les taux par âge, par régime d'étude et par niveau d'enseignement. C'est en fait la résultante de tous les taux de scolarisation, aussi dits de fréquentation, traduits en durée moyenne d'études pour l'ensemble d'une génération.

Ce résultat doit évidemment être analysé en fonction des facteurs les plus déterminants de la scolarisation. Il y a, en premier lieu, l'accès aux études à différents niveaux, puis les durées théorique et effective de ces études. Le nombre d'années que doit durer un cycle d'études agit directement sur les taux de scolarisation. Il faut aussi considérer qu'une partie de ceux et celles qui entreprennent un programme d'études ne le terminent pas et que ce phénomène influe aussi sur la durée moyenne des études, puisque diplômés et décrocheurs n'ont habituellement pas la même durée d'études. Par contre, un phénomène comme le redoublement ou, aux niveaux postsecondaires, les passages d'un programme à l'autre agissent dans le même sens qu'une persévérance accrue et tendent à allonger le temps de scolarisation.

C'est l'interaction de ces divers aspects de la scolarisation qu'illustre le présent texte, dans la mesure où les données nécessaires au calcul des indices sont disponibles et accessibles.

\section{L'ESPÉRANCE DE SCOLARISATION}

Une des multiples facettes de la scolarisation, et sans doute la plus synthétique, est le nombre moyen d'années pendant lesquelles une génération réelle ou fictive aura fréquenté un établissement scolaire.

Au Quêbec, cette durée de la fréquentation scolaire est de 15,28 ans en 1992-1993 (tableau 1). Depuis 1987-1988, elle a crû de 0,9 an, soit l'équivalent de 0,1 à 0,4 an de scolarité supplémentaire chaque année. Cette durée, aussi dénommée espérance de scolarisation ou de vie scolaire, est comparable à celle qu'on observe en France. 
Techniquement, l'espérance de scolarisation pour une année scolaire donnée est égale à la somme des taux de scolarisation (ou de fréquentation) à temps complet (ou l'équivalent) par année d'âge. Un taux de scolarisation équivaut à un nombre moyen d'années de scolarisation par personne. La somme des taux par âge indique la durée hypothétique des études d'une génération qui serait soumise pendant toute son existence aux taux observés durant une seule année.

Dans son Rapport mondial sur l'éducation publié en 1993, l'UNESCO rapporte une estimation sommaire de cet indicateur pour environ 70 pays en 1990. Le Canada figure au premier rang mondial, avec une espérance de 16 ans ${ }^{2}$. On trouvera au tableau 2 les valeurs les plus élevées et les plus faibles observées dans les pays pour lesquels l'UNESCO disposait d'une information suffisante.

\section{L'espérance de scolarisation et l'organisation scolaire}

$\mathrm{Au}$ Québec, la répartition par ordre d'enseignement des années de scolarité moyennes fait ressortir que tous les gains récents sont attribuables, soit à l'éducation des adultes, soit à l'enseignement post-secondaire. La durée normale des études primaires et secondaires, de 11 ans, correspond à la période de fréquentation scolaire obligatoire, qui va de l'âge de 6 ans à l'âge de 16 ans. À ces niveaux, l'espérance de scolarisation correspond depuis assez longtemps à la durée théorique des êtudes. Cela n'a rien d'êtonnant quand on sait que l'accès à ces ordres d'enseignement est, en fait, universel. Puisque tous les jeunes passent en pratique par les études primaires, il est normal de constater qu'ils y restent en moyenne 6 ans. Au secondaire, une espérance de 5 ans, sous des dehors anodins, peut dissimuler les effets de phénomènes contradictoires comme ceux du retard scolaire et de l'abandon, qui tendent, l'un à augmenter la durée des études, l'autre à la réduire.

L'enseignement collégial est d'une durée de deux ans pour les élèves inscrits à la formation préuniversitaire et de trois ans pour les élèves inscrits à la formation technique. La durée probable de séjour à ce niveau d'études dans l'un et l'autre type

2 Les hypothèses de conversion de l'enseignement à temps partiel en équivalents temps plein (ETP) semblent toutefois agir légèrement dans le sens d'une surestimation du temps de scolarisation. En outre, les données pour la France ne correspondent pas à celles de la publication française. 
TABLEAU 1 - Espérance de scolarisation à l'ãge de 6 ans au Québec et en France (en années)

\begin{tabular}{lrrrrrr}
\hline & $1987-$ & $1988-$ & $1989-$ & $1990-$ & $1991-$ & $1992-$ \\
& 1988 & 1989 & 1990 & 1991 & 1992 & 1993 \\
\hline Primaire (jeunes) & 6,11 & 6,12 & 6,12 & 6,11 & 6,10 & 6,08 \\
Secondaire (jeunes) & 5,09 & 5,03 & 4,97 & 4,95 & 4,93 & 5,07 \\
Secteur des adultes & 0,30 & 0,23 & 0,58 & 0,61 & 0,85 & 0,79 \\
$\quad$ Sous-total & 11,50 & 11,39 & 11,67 & 11,67 & 11,88 & 11,94 \\
Collégial a & 1,55 & 1,55 & 1,55 & 1,59 & 1,68 & 1,75 \\
Universitaire & 1,27 & 1,33 & 1,39 & 1,46 & 1,53 & 1,59 \\
$\quad$ Toral (Québec) & 14,32 & 14,27 & 14,61 & 14,72 & 15,09 & 15,28 \\
\hline$\quad$ France & 14,2 & 14,4 & 14,6 & 14,8 & 15,0 & 15,2 \\
\hline
\end{tabular}

Sources : ministère de l'Éducation du Québec et ministère de l'Éducation nationale (France).

a. Au collêgial, seul l'enseignement ordinaire à temps complet a été pris en considération. Les inscriptions à temps partiel et les inscriptions dénombrées à l'enseignement des adultes ne sont pas transformées en équivalents temps plein (ETP).

de formation est de 1,75 an en 1992-1993 pour toute personne "susceptible" de fréquenter un établissement collégial. Cette durée probable est inférieure à la durée théorique, principalement parce que tout le monde ne poursuit pas des études à ce niveau. Le même genre de situation existe à l'enseignement universitaire, où on distingue trois cycles d'études. Le premier conduit au baccalauréat, le deuxième à la maîtrise et le troisième au doctorat. Le premier cycle dure normalement trois ans, le deuxième deux et le troisième trois ans. Les universités décernent aussi des certificats et diplômes pour sanctionner la réussite de programmes courts. Pour l'ensemble des trois

TABLEAU 2 - Estimation de l'espérance de vie scolaire dans certains pays en 1990

Les cinq pays où elle est la plus faible ${ }^{\mathrm{a}}$

$\begin{array}{ll}\text { Niger } & 2,1 \\ \text { Guinée } & 2,7 \\ \text { Bangladesh } & 5,0 \\ \text { Rwanda } & 5,7 \\ \text { Maroc } & 6,3\end{array}$

Les cinq pays où elle est la plus élevée a

$\begin{array}{ll}\text { France } & 14,5 \\ \text { Nouvelle-Zélande } & 14,6 \\ \text { Norvège } & 14,6 \\ \text { Ettats-Unis } & 15,4 \\ \text { Canada } & 16,0\end{array}$

Source : UNESCO.

a. Parmi les pays pour lesquels l'UNESCO dispose de données pertinentes. 
TABLEAU 3 - Espérance de scolarisation à l'âge de 6 ans selon le sexe (en années)

\begin{tabular}{lrrrrrr}
\hline & $1987-$ & $1988-$ & $1989-$ & $1990-$ & $1991-$ & $1992-$ \\
& 1988 & 1989 & 1990 & 1991 & 1992 & 1993 \\
\hline Sexe masculin & & & & & & \\
Primaire (jeunes) & 6,16 & 6,17 & 6,17 & 6,17 & 6,18 & 6,15 \\
Secondaire (jeunes) & 5,11 & 5,04 & 4,98 & 4,96 & 4,93 & 5,10 \\
Secteur des adultes & 0,29 & 0,25 & 0,59 & 0,63 & 0,85 & 0,78 \\
$\quad$ Sous-total & 11,56 & 11,46 & 11,74 & 11,76 & 11,96 & 12,03 \\
Collègial & 1,36 & 1,35 & 1,35 & 1,37 & 1,46 & 1,54 \\
Universitaire & n.d. & 1,20 & 1,11 & 1,29 & 1,36 & 1,40 \\
$\quad$ Toral & n.d. & 14,01 & 14,20 & 14,42 & 14,78 & 14,97 \\
Sexe féminin & & & & & & \\
Primaire (jeunes) & 6,06 & 6,08 & 6,07 & 6,05 & 6,03 & 6,00 \\
Secondaire (jeunes) & 5,07 & 5,02 & 4,96 & 4,94 & 4,93 & 5,04 \\
Secteur des adultes & 0,31 & 0,21 & 0,57 & 0,60 & 0,86 & 0,79 \\
$\quad$ Sous-total & 11,44 & 11,31 & 11,60 & 11,59 & 11,82 & 11,83 \\
Collégial & 1,74 & 1,75 & 1,76 & 1,81 & 1,90 & 1,97 \\
Universitaire & n.d. & 1,46 & 1,68 & 1,63 & 1,71 & 1,77 \\
$\quad$ Toral & n.d. & 14,52 & 15,04 & 15,03 & 15,43 & 15,57 \\
\hline
\end{tabular}

Source : ministère de l'Éducation du Quëbec.

cycles, y compris les programmes courts, l'espérance de scolarisation est de 1,59 an en 1992-1993.

\section{L'espérance de scolarisation selon le sexe et les redoublements}

On a une meilleure idée de l'impact du retard scolaire en examinant les résultats selon le sexe au primaire et au secondaire (tableau 3). À ces niveaux, la scolarisation des garçons semble légèrement supérieure à celle des filles si on ne tient compte que de la durée, mais on sait pertinemment que les filles réussissent mieux et plus vite; c'est seulement parce qu'elles subissent moins de retard qu'elles ont une scolarité plus courte. On trouvera au tableau 4 une évaluation du temps perdu à cause des redoublements au primaire et au secondaire général du secteur des jeunes. Ce résultat est facile à obtenir si la proportion des élèves redoublant une classe est connue; il suffit d'appliquer cette proportion à l'espérance de scolarisation calculée pour la population correspondante ${ }^{3}$.

3 La procédure complète pour obtenir le mème résultat consisterait à faire la somme des taux de scolarisation par âge ayant comme numérateur les élèves qui redoublent une classe. 
TABLEAU 4 - Proportions de redoublements (en pourcentage) et durée de scolarisation (en années) consacrée aux redoublements à l'enseignement primaire et secondaire général au secteur des jeunes

\begin{tabular}{lllllll}
\hline & $1987-$ & $1988-$ & $1989-$ & $1990-$ & $1991-$ & $1992-$ \\
1988 & 1989 & 1990 & 1991 & 1992 & 1993 \\
\hline
\end{tabular}

Primaire

$\begin{array}{lllllll}\text { Garçons } & 6,0 & 6,1 & 6,4 & 7,0 & 6,4 & 6,4 \\ \text { Filles } & 3,7 & 3,8 & 4,1 & 4,4 & 4,0 & 4,0 \\ \text { Ensemble } & 4,9 & 5,0 & 5,3 & 5,7 & 5,3 & 5,3\end{array}$

Secondaire (général)

$\begin{array}{lrrrrrr}\text { Garçons } & 11,2 & 11,7 & 11,9 & 12,3 & 12,2 & 11,7 \\ \text { Filles } & 7,2 & 7,4 & 7,7 & 7,8 & 7,7 & 7,3 \\ \text { Ensemble } & 9,2 & 9,6 & 9,9 & 10,0 & 10,0 & 9,5\end{array}$

Primaire

$\begin{array}{lllllll}\text { Garçons } & 0,37 & 0,38 & 0,39 & 0,43 & 0,40 & 0,39 \\ \text { Filles } & 0,22 & 0,23 & 0,25 & 0,27 & 0,24 & 0,24 \\ \text { Ensemble } & 0,30 & 0,31 & 0,32 & 0,35 & 0,32 & 0,32\end{array}$

Secondaire ${ }^{a}$

$\begin{array}{lllllll}\text { Garçons } & 0,57 & 0,59 & 0,59 & 0,61 & 0,60 & 0,60 \\ \text { Filles } & 0,37 & 0,37 & 0,38 & 0,39 & 0,38 & 0,37 \\ \text { Ensemble } & 0,47 & 0,48 & 0,49 & 0,50 & 0,49 & 0,48\end{array}$

Total (primaire et secondaire)

$\begin{array}{lllllll}\text { Garçons } & 0,94 & 0,97 & 0,98 & 1,04 & 1,00 & 0,99 \\ \text { Filles } & 0,59 & 0,60 & 0,63 & 0,66 & 0,62 & 0,61 \\ \text { Ensemble } & 0,77 & 0,79 & 0,81 & 0,85 & 0,81 & 0,80\end{array}$

Source : ministère de l'Éducation du Québec.

a. C'est la proportion de redoublement mesurée au général qui est appliquée à l'ensemble de la scolarisation secondaire (générale et professionnelle).

Il en va autrement à l'enseignement post-secondaire, où l'espérance de scolarisation est significativement inférieure à la durée théorique des études. C'est aussi là qu'apparaît la grande différence entre la scolarisation des hommes et celle des femmes; de 1988-1989 à 1992-1993, pour l'ensemble du collégial et de l'universitaire, l'espérance des femmes oscille entre 3,21 ans et 3,74 ans comparativement à des valeurs comprises entre 2,46 (en 1989-1990) et 2,94 pour les hommes. Ce n'est évidemment pas l'efficacité qui explique la faible 
scolarisation des hommes à ce niveau; c'est plutôt la probabilité moindre d'entreprendre des études supérieures, comme on le verra dans la section portant sur l'accès aux ètudes.

Ce qui précède met en lumière deux composantes fondamentales de la scolarisation, peu importe la forme sous laquelle elle est mesurée, soit la proportion de personnes qui accèdent à un ordre d'enseignement et le nombre d'années que ces personnes y passent. En d'autres mots, on peut dire que, si le tiers des personnes accèdent à l'université et y restent trois ans, le résultat (strictement en nombre moyen d'années de scolarisation par personne) est le même que si tout le monde accède à l'enseignement universitaire mais pendant une seule année.

L'espérance de scolarisation ne renseigne pas sur tous les aspects de la fréquentation scolaire mais elle est son expression la plus synthétique car elle tient compte de l'accès aux études et de la durée effective de celles-ci, qui dépendent à leur tour des abandons et des retards. Cette mesure doit être mise en perspective avec d'autres indicateurs qui ont un impact plus ou moins direct sur elle, soit la probabilité d'accès aux études de même que la durée effective des études pour les personnes qui s'y engagent.

\section{L'ACCÈS AUX ÉTUDES}

Sachant quelle est la moyenne de scolarité par personne, nous nous demanderons comment se répartit cette moyenne ou plus exactement quelle est la proportion des personnes qui accèdent aux études et qui, de ce fait, génèrent les moyennes observées plus haut (comme si, après avoir déterminé le PIB par habitant, on voulait savoir quelle proportion de la population a contribué au PIB). On verra quel lien existe entre l'espérance de scolarisation et la durée moyenne des études pour ceux et celles qui entreprennent ces études.

\section{L'accès aux êtudes selon le niveau}

$\mathrm{Au}$ primaire et au secondaire, on l'a vu, l'accès est quasi universel ${ }^{4}$ et il en découle que les durées moyennes exprimées par l'espérance de scolarisation sont très voisines de la durée

4 Certains jeunes restent en marge du système scolaire officiel, étant dans un centre d'accueil, dans une école qui n'est pas reconnue légalement ou à la maison. 
TABLEAU 5 - Probabilité d'accès aux divers ordres et cycles d'enseignement, sexes réunis (en pourcentage)

\begin{tabular}{lrrrrrr}
\hline & $1987-$ & $1988-$ & $1989-$ & $1990-$ & $1991-$ & $1992-$ \\
& 1988 & 1989 & 1990 & 1991 & 1992 & 1993 \\
\hline Secondaire & 98,7 & 99,0 & 99,0 & 99,6 & 98,3 & 98,6 \\
Collégial a & 56,9 & 57,6 & 58,7 & 60,4 & 61,2 & 63,8 \\
Universitaire & & & & & & \\
$\quad$ Baccalauréat & 34,6 & 34,8 & 35,6 & 36,8 & 37,9 & 39,4 \\
$\quad$ Maîtrise & 7,3 & 6,9 & 6,8 & 7,1 & 7,8 & 8,3 \\
$\quad$ Doctorat & 1,4 & 1,3 & 1,5 & 1,5 & 1,7 & 1,8 \\
\hline
\end{tabular}

Source : ministère de l'Éducation du Guébec.

a. Il s'agit de l'accès aux programmes de l'enseignement ordinaire menant à un diplôme d'études collégiales (DEC).

théorique. Au collégial, on perçoit mieux l'action de l'accessibilité sur l'espérance de scolarisation. La durée des études y est théoriquement de deux ou de trois ans selon le type de formation; on observe cependant que l'espérance de scolarisation n'atteint que 1,75 année. Or, en 1992-1993, l'accès à l'enseignement collégial est de $63,8 \%$ (tableau 5). En supposant que ces deux dernières valeurs soient stables dans le temps, on pourrait considérer que le rapport $1,75 / 0,638=2,74$ est la mesure de la durée des études de ceux qui entreprennent des études collêgiales. Le même calcul avec les données de 1987-1988 (1,55/0,569) aboutit à une durée de 2,72 années. On peut donc déjà conclure que la croissance de l'espérance de scolarisation observée au collégial résulte presque essentiellement de la croissance de l'accès à cet enseignement. On parvient à la même conclusion en comparant la croissance de l'espérance de scolarisation et la croissance de la probabilité d'entreprendre des études collégiales, qui se sont accrues respectivement de $14,0 \%$ et de $12,3 \%$ par rapport à leur niveau de 1987-1988. Les progrès observés sont sans doute annonciateurs d'une autre poussée de la demande d'accès aux études universitaires pendant les années à venir.

Dans l'enseignement universitaire, les progrès sont du même ordre de grandeur. Au baccalauréat, l'accès a connu une augmentation relative de $14 \%$ depuis 1987-1988; en 1992 1993, 39,4\% des Québécois et Québécoises entreprennent un programme de baccalauréat, contre $34,6 \%$ cinq ans plus tôt. La progression relative aux cycles supérieurs a aussi été 
rapide, même si encore peu de gens parviennent aux études de maitrise ou de doctorat.

\section{L'accès aux études selon le sexe et le niveau}

Il faut encore porter une attention particulière aux résultats selon le sexe aux niveaux post-secondaires (tableau 6). Au collégial, l'évolution de l'accès, du même ordre pour les hommes et pour les femmes, correspond aussi à l'évolution de la scolarisation, signe que la durée effective des études a peu changé. À l'université, cependant, on est frappé par le fait que, depuis 1987-1988, la croissance de l'accès aux études est beaucoup plus rapide pour les femmes que pour les hommes. Entre 1987-1988 et 1992-1993, la croissance de l'accès a été de 3,9 points au baccalauréat et de 0,8 point à la maîtrise pour les hommes, de 5,6 points et de 1,0 point pour les femmes.

TABLEAU 6 - Probabilité d'accès aux divers ordres et cycles d'enseignement, selon le sexe (en pourcentage)

\begin{tabular}{lcccccc}
\hline & $1987-$ & $1988-$ & $1989-$ & $1990-$ & $1991-$ & $1992-$ \\
& 1988 & 1989 & 1990 & 1991 & 1992 & 1993 \\
\hline $\begin{array}{l}\text { Secondaire } \\
\text { Sexe masculin }\end{array}$ & 98,9 & 99,3 & 99,3 & 99,9 & 98,2 & 98,6 \\
$\quad$ Sexe féminin & 98,5 & 98,7 & 98,7 & 99,3 & 98,4 & 98,7 \\
Collégial a & & & & & & \\
$\quad$ Sexe masculin & 49,9 & 50,3 & 50,8 & 52,4 & 54,0 & 56,5 \\
$\quad$ Sexe féminin & 64,2 & 65,1 & 66,9 & 68,4 & 68,7 & 71,6 \\
Universitaire & & & & & & \\
Baccalauréat & & & & & & \\
$\quad$ Sexe masculin & 30,6 & 31,0 & 31,7 & 32,3 & 33,3 & 34,5 \\
$\quad$ Sexe féminin & 38,8 & 38,8 & 39,5 & 41,4 & 42,5 & 44,5 \\
Maitrise & & & & & & \\
$\quad$ Sexe masculin & 7,4 & 7,0 & 6,9 & 7,1 & 7,8 & 8,4 \\
$\quad$ Sexe féminin & 7,2 & 6,8 & 6,6 & 7,1 & 7,8 & 8,1 \\
Doctorat & & & & & & \\
$\quad$ Sexe masculin & 1,7 & 1,6 & 1,8 & 1,9 & 2,0 & 2,2 \\
$\quad$ Sexe féminin & 1,0 & 1,0 & 1,1 & 1,1 & 1,3 & 1,4 \\
\hline
\end{tabular}

Source : ministère de l'Éducation du Québec

a. Il s'agit de l'accès aux programmes de l'enseignement ordinaire menant à un diplôme d'études collégiales (DEC). 


\section{LA DURÉE DES ÉTUDES}

On vient d'avoir un aperçu de la principale composante de la scolarisation, soit l'accès aux études. Il s'agit certes du facteur le plus facile à percevoir, de celui qui joue à l'entrée. Il tombe sous le sens que ce qui se produit à la sortie influencera aussi la durée, mais de manière un peu plus complexe. Ce n'est plus la quantité de sortants qui aura un impact puisqu'elle est la même que celle des entrants et que leur rôle est déjà escompté. Ce sera plutôt le moment où les personnes quitteront les études, c'est-à-dire le calendrier des sorties. À ce sujet, nous distinguerons deux catégories, soit les diplômes et les interruptions ou sorties sans diplôme.

D'un point de vue théorique, les relations entre le calendrier des sorties, l'accès aux études et l'espérance de scolarisation peuvent s'exprimer par la formule suivante ${ }^{5}$ :

$$
\mathrm{E}(\mathrm{s})=\mathrm{A}(\mathrm{s}) \times[\mathrm{T}(\mathrm{d}) \times \mathrm{R}+\mathrm{T}(\mathrm{a}) \times(1-\mathrm{R})]
$$

où les éléments sont :

$\mathrm{E}(\mathrm{s})$ : l'espérance de scolarisation;

$\mathrm{A}(\mathrm{s})$ : la proportion des personnes qui accèdent aux études;

T(d) : la durée des études pour les personnes diplômées;

$\mathrm{T}(\mathrm{a})$ : la durée des études avant l'abandon;

$\mathrm{R}$ : la proportion de diplômés parmi ceux qui accèdent aux études.

Dans le membre droit de l'équation, la partie entre crochets correspond à la durée moyenne effective des études de tous les sortants et peut être désignée par T(s). La forme générale de l'équation précédente devient donc:

$$
\mathrm{E}(\mathrm{s})=\mathrm{A}(\mathrm{s}) \times \mathrm{T}(\mathrm{s}) .
$$

Cependant, les données chiffrées les plus courantes ayant trait au calendrier de sortie des études ne décrivent pas la durée des études comme telle. Le tableau A, en annexe, présente des taux de réussite et de persévérance à diverses durées dans des cohortes identifiées par l'année de la première inscription. Ce type d'information rejoint certes le sujet de la durée des études mais ne peut fournir aucun des éléments de l'équation présentée au paragraphe précédent. Au tableau B,

5 On pourrait ajouter au membre de droite la durée correspondant aux personnes qui n'ont pas entrepris d'études; elle est égale à zéro, soit : $\{[1-A(s)] \times 0\}$. 
toujours en annexe, on trouve aussi des probabilités d'accès aux diplômes; il s'agit d'indicateurs apparentés au sujet principal, mais d'où la notion de durée est encore absente.

\section{La durêe "transversale" ou "intrinsèque"}

On a vu, dans la section consacrée à l'accès aux études, un exemple de calcul "transversal" de la durée au sujet de l'enseignement collégial. Nous avions obtenu ce résultat en modifiant ainsi la formule précédente :

$$
T(s)=E(s) \div A(s) \text {. }
$$

L'application de cette formule à des données portant sur une seule année produit une mesure théorique de la durée, soit celle qu'on observerait dans une cohorte si les autres termes de l'équation (l'espérance de scolarisation et la probabilité d'accès aux études) demeuraient stables pendant une période suffisamment longue. C'est la raison pour laquelle on ne peut parler ici de durée réelle, mais de durée "intrinsèque". Le calcul de cette durée peut être effectué pour le secondaire et pour le collégial; les résultats ${ }^{6}$ apparaissent au tableau 7 .

\section{La durée "longitudinale"}

Le tableau 7 présente une information concernant l'ensemble des personnes inscrites à un ordre d'enseignement ou à un programme de formation mais ne distingue pas celles qui obtiennent un diplôme de celles qui abandonnent leurs études. Cette distinction est impossible lorsqu'on utilise des données transversales pour la simple raison qu'on ne sait pas d'avance quelle sera l'issue des études pour des personnes qui sont toutes inscrites sur le même pied au même moment. Dans l'équation générale précédente, l'espérance de scolarisation est reliée à la durée des études avant le diplôme [T(d)], à la durée des études avant l'abandon [T(a)] et à la proportion de diplômés (R); ces trois quantités sont cependant absentes des calculs produisant les résultats du tableau 7 .

6 Dans le cas de l'enseignement universitaire, l'espérance de scolarisation [E(s)] n'est pas ventilée par niveau de formation (baccalauréat, maitrise, doctorat), alors que les probabilités d'accès aux études [A(s)] sont calculées par niveau de formation. 
TABLEAU 7 - Durée (transversale) des études (en années de scolarité)

\begin{tabular}{|c|c|c|c|c|c|c|}
\hline & $\begin{array}{l}1987- \\
1988\end{array}$ & $\begin{array}{l}1988- \\
1989\end{array}$ & $\begin{array}{l}1989- \\
1990\end{array}$ & $\begin{array}{l}1990- \\
1991\end{array}$ & $\begin{array}{l}1991- \\
1992\end{array}$ & $\begin{array}{l}1992- \\
1993\end{array}$ \\
\hline \multicolumn{7}{|l|}{ Enseignement secondaire } \\
\hline Sexes rêunis & 5,16 & 5,08 & 5,02 & 4,97 & 5,02 & 5,14 \\
\hline Sexe masculin & 5,17 & 5,08 & 5,02 & 4,96 & 5,02 & 5,17 \\
\hline Sexe fêminin & 5,15 & 5,09 & 5,03 & 4,97 & 5,01 & 5,11 \\
\hline \multicolumn{7}{|l|}{ Enseignement collégial } \\
\hline \multicolumn{7}{|l|}{ Sexes réunis } \\
\hline Toutes formations & 2,72 & 2,69 & 2,64 & 2,63 & 2,75 & 2,74 \\
\hline Préuniversitaire & 2,20 & 2,17 & 2,14 & 2,12 & 2,17 & 2,13 \\
\hline Technique & 3,70 & 3,71 & 3,69 & 3,60 & 3,79 & 3,86 \\
\hline \multicolumn{7}{|l|}{ Sexe masculin } \\
\hline Toutes formations & 2,73 & 2,68 & 2,66 & 2,61 & 2,70 & 2,73 \\
\hline Préuniversitaire & 2,26 & 2,20 & 2,18 & 2,15 & 2,18 & 2,17 \\
\hline Technique & 3,68 & 3,74 & 3,74 & 3,57 & 3,67 & 3,71 \\
\hline \multicolumn{7}{|l|}{ Sexe féminin } \\
\hline Toutes formations & 2,71 & 2,69 & 2,63 & 2,65 & 2,77 & 2,75 \\
\hline Préuniversitaire & 2,16 & 2,14 & 2,10 & 2,10 & 2,16 & 2,11 \\
\hline Technique & 3,69 & 3,69 & 3,64 & 3,64 & 3,89 & 4,00 \\
\hline
\end{tabular}

Source : ministère de l'Éducation du Guébec.

Le recours aux données longitudinales est indispensable pour obtenir une mesure distincte des quantités T(d) et T(a). C'est au moment où les inscrits quittent leurs études que les diplômes et les abandons peuvent être différenciés; il devient alors possible d'évaluer rétrospectivement le temps consacré à la scolarisation pour chacune des catégories de sortants ${ }^{7}$.

Le tableau 8 offre des résultats obtenus de façon longitudinale. C'est seulement au secondaire que les mesures sont rétrospectives et incluent les personnes qui ont quitté leurs études sans diplôme. Le rapprochement entre les données des tableaux 7 et 8 ne peut donc se faire que pour le secondaire, mais il ne faut pas perdre de vue que les valeurs du tableau 7 portent sur une seule année scolaire et sur plusieurs cohortes à la fois tandis que les valeurs du tableau 8 portent sur une

7 On peut aussi effectuer le calcul de la durée de façon prospective en suivant une cohorte de premières inscriptions jusqu'à son extinction. Cette procédure, retenue pour l'enseignement collégial, présente l'inconvénient de nous obliger à nous référer à une année ancienne pour offrir des résultats définitifs. 
TABLEAU 8 - Durée (longitudinale) des études

\begin{tabular}{|c|c|c|c|c|c|}
\hline $\begin{array}{l}\text { ENSEIGNEMENT } \\
\text { SECONDAIRE }\end{array}$ & \multicolumn{5}{|c|}{$\begin{array}{l}\text { Durée en années de scolarité selon } \\
\text { l'année de sortie }\end{array}$} \\
\hline & 1989 & 1990 & 1991 & 1992 & 1993 \\
\hline \multicolumn{6}{|l|}{ Sexes réunis } \\
\hline Sortie avec diplôme & 5,17 & 5,18 & 5,19 & 5,16 & 5,16 \\
\hline Sortie sans diplôme & 4,29 & 4,26 & 4,23 & 4,16 & 4,35 \\
\hline Total & 4,89 & 4,93 & 4,96 & 4,96 & 4,96 \\
\hline \multicolumn{6}{|l|}{ Sexe masculin } \\
\hline Sortie avec diplôme & 5,20 & 5,23 & 5,24 & 5,21 & 5,21 \\
\hline Sortie sans diplôme & 4,29 & 4,25 & 4,27 & 4,21 & 4,40 \\
\hline Total & 4,87 & 4,91 & 4,96 & 4,97 & 4,97 \\
\hline \multicolumn{6}{|l|}{ Sexe fëminin } \\
\hline Sortie avec diplôme & 5,13 & 5.14 & 5,14 & 5,11 & 5,12 \\
\hline Sortie sans diplôme & 4,27 & 4,28 & 4,18 & 4,08 & 4,26 \\
\hline Total & 4,92 & 4,95 & 4,95 & 4,95 & 4,96 \\
\hline $\begin{array}{l}\text { ENSEIGNEMENT } \\
\text { COLLÉGIAL }\end{array}$ & \multicolumn{5}{|c|}{$\begin{array}{l}\text { Nombre de trimestres à temps plein } \\
\text { selon l'année de la première inscription }\end{array}$} \\
\hline & 1980 & 1983 & 1986 & 1987 & 1988 \\
\hline \multicolumn{6}{|l|}{ Préuniversitaire } \\
\hline $\begin{array}{l}\text { Avec diplôme } \\
\text { Sans diplôme }\end{array}$ & 5,1 & 5,2 & $\begin{array}{r}5,2 \\
\text { n. d. }\end{array}$ & 5,1 & 4,9 \\
\hline \multicolumn{6}{|l|}{ Technique } \\
\hline $\begin{array}{l}\text { Avec diplôme } \\
\text { Sans diplôme }\end{array}$ & 6,5 & 6,7 & $\begin{array}{l}6,7 \\
\text { n.d. }\end{array}$ & 6,6 & 6,5 \\
\hline $\begin{array}{l}\text { ENSEIGNEMENT } \\
\text { UNIVERSITAIRE }\end{array}$ & \multicolumn{5}{|c|}{$\begin{array}{l}\text { Nombre moyen a de trimestres selon } \\
\text { l'année de sortie }\end{array}$} \\
\hline & 1989 & 1990 & 1991 & 1992 & 1993 \\
\hline \multicolumn{6}{|l|}{ Avec diplôme } \\
\hline Baccalauréat & 9,0 & 9,3 & 9,5 & 9,4 & n.d. \\
\hline Maîtrise & n.d. & n.d. & 9,0 & 9,0 & n.d. \\
\hline Doctorat & n.d. & n.d. & 15,1 & 15,5 & n.d. \\
\hline Sans diplôme & & & n.d. & & \\
\hline
\end{tabular}

Source : ministère de l'Éducation du Québec.

a. Y compris les interruptions.

seule cohorte et sur plusieurs années d'observation. Si on laisse de côté la durée transversale déterminée en 1992-1993 (5,14 années), on peut voir que la durée moyenne des études des sortants du secondaire de 1991 à 1993 (4,96 années) est passablement similaire aux durées transversales déterminées en 1990-1991 (4,97 années) et en 1991-1992 (5,02 années). 


\section{CONSIDÉRATIONS SUR LES DEUX APPROCHES}

Nonobstant les différences entre les résultats des deux approches, le calcul transversal de la durée moyenne découle aisément du calcul de l'espérance de scolarisation et de la probabilité d'accès aux études, deux éléments déjà connus. La difficulté de l'approche transversale est d'ordre épistémologique puisqu'il faut accepter l'idée qu'une durée puisse être mesurée sur la base de l'expérience de plusieurs cohortes observées à un instant précis, comme une dérivée mesure la pente d'une courbe à un point particulier. En outre, le niveau de détail possible est limité puisque la distinction entre diplômés et sortants sans diplôme n'est pas possible transversalement.

À l'opposé, dans l'approche longitudinale, la notion de durée se perçoit spontanement mais nécessite un calcul plus ardu à partir de données souvent difficiles d'accès. L'absence actuelle d'information sur les personnes qui sortent sans diplôme du collégial et de l'université illustre cette difficulté, en même temps qu'elle témoigne peut-être d'un manque d'attention, car actuellement les regards ne se tournent que vers le segment de la population scolaire qui obtient un diplôme. Le traitement longitudinal peut enrichir le portrait de la scolarisation en permettant de distinguer la scolarité "consommée" et la scolarité "manquante". N'est-ce pas par un examen de toutes les sorties du système d'éducation - avec et sans diplôme qu'on sera le mieux en mesure d'en évaluer l'efficacité ?

\section{RÉFÉRENCES BIBLIOGRAPHIgUES}

FRANCE, 1994. L'État de l'école, 30 indicateurs sur le système éducatif. Ministère de l'Éducation nationale, Direction de l'évaluation et de la prospective, no 4, octobre, $77 \mathrm{p}$.

LESPÉRANCE, André, 1980. La Fréquentation scolaire postsecondaire au Québec et en Ontario de 1976-1977 à 1978-1979 et aux ÉtatsUnis en 1976-1977. Guébec, Ministère de l'Éducation, Direction des études économiques et démographiques, $77 \mathrm{p}$.

MINISTĖRE DE L'ÉDUCATION, 1995. Indicateurs de l'éducation, Édition 1994. Québec, Gouvernement du Québec, 112 p.

UNESCO, 1993. Rapport mondial sur l'éducation 1993, Paris, 172 p. 


\section{ANNEXE}

Tableau A - Proportion (en pourcentage) des nouveaux inscrits diplômés et inscrits sans diplôme selon l'année de la première inscription, le type de programme et l'ordre d'enseignement en fonction de la durée (en années) écoulée depuis le début des études

\begin{tabular}{|c|c|c|c|c|c|c|c|c|}
\hline \multirow[b]{2}{*}{ SECONDAIRE } & \multicolumn{4}{|c|}{$\begin{array}{l}\text { Proportion de diplómés } \\
\text { (\%) selon la durée }\end{array}$} & \multicolumn{4}{|c|}{$\begin{array}{l}\text { Proportion d'inscrits non } \\
\text { diplômés (\%) selon la durée }\end{array}$} \\
\hline & 5 & 6 & 7 & & 4 & 5 & 6 & \\
\hline $1985-1986$ & 54,8 & 63,8 & 67,8 & & 88,1 & 27,1 & 15,6 & \\
\hline $1986-1987$ & 56,3 & 65,8 & 69,5 & & 89,9 & 28,0 & 15,3 & \\
\hline $1987-1988$ & 59,2 & 67,7 & 71,4 & & 91,6 & 27,4 & & \\
\hline 1988-1989 & 57,7 & 67,8 & & & 92,7 & & & \\
\hline COLLÉGIAL & 2 & 3 & 4 & 5 & 2 & 3 & 4 & 5 \\
\hline \multicolumn{9}{|c|}{ Préuniversitaire } \\
\hline $1986-1987$ & 30,7 & 52,9 & 61,2 & 65,2 & 44,6 & 18,7 & 9,9 & \\
\hline $1987-1988$ & 34,1 & 56,7 & 64,9 & 69,0 & 43,6 & 18,3 & 10,6 & \\
\hline 1988-1989 & 33,9 & 56,0 & 64,5 & 68,6 & 44,1 & 20,4 & 11,7 & \\
\hline $1989-1990$ & 34,6 & 56.6 & 64,3 & & 44,4 & 20,4 & & \\
\hline \multicolumn{9}{|l|}{ Technique } \\
\hline $1983-1984$ & & 30,6 & 46,8 & 53,0 & 69,4 & 33,5 & 13,6 & \\
\hline $1986-1987$ & & 25,4 & 40,8 & 46,9 & 65,6 & 32,9 & 14,8 & \\
\hline $1987-1988$ & & 28.7 & 44,8 & 50,7 & 67,6 & 33,2 & 15,5 & \\
\hline $1988-1989$ & & 29,4 & 45,7 & 51,5 & 69,5 & 34,9 & 15,7 & \\
\hline UNIVERSTTÉ & 3 & 4 & 5 & 6 & 3 & 4 & 5 & 6 \\
\hline \multicolumn{9}{|l|}{ Baccalauréat } \\
\hline $1986-1987$ & 25,3 & 48,5 & 57,5 & 60,2 & 40,4 & 16,2 & 7,2 & 4,3 \\
\hline $1987-1988$ & 25,9 & 49,4 & 57,9 & & 39,7 & 15,5 & 6,9 & \\
\hline 1988-1989 & 26,6 & 50,5 & 60,0 & & 41,6 & 16,3 & & \\
\hline 1989-1990 & 26,5 & & & & 41,4 & & & \\
\hline \multicolumn{9}{|l|}{ Maîtrise } \\
\hline 1986-1987 & 28,5 & 38,2 & 45,8 & 50,1 & 32,1 & 18,2 & 8,9 & 4,5 \\
\hline 1987-1988 & 33,6 & 45,2 & 52,8 & & 33,9 & 19,8 & 9,8 & \\
\hline 1988-1989 & 35,5 & 48,0 & 56,0 & & 33,7 & 17,0 & & \\
\hline 1989-1990 & 39,7 & & & & 31,5 & & & \\
\hline Doctorat & 4 & 5 & 6 & 7 & 4 & 5 & 6 & 7 \\
\hline 1985-1986 & 14,0 & 23,9 & 33,7 & 41,5 & 51,3 & 38,4 & 27,4 & 15,3 \\
\hline 1986-1987 & 16,2 & 28,0 & 36,4 & & 54,0 & 38,7 & 27.3 & \\
\hline $1987-1988$ & 16,9 & 29,8 & & & 53,9 & 37,7 & & \\
\hline 1988-1989 & 12,9 & & & & 55,6 & & & \\
\hline
\end{tabular}

Source : ministère de l'Éducation du Québec. 
Tableau B - Probabilité d'accès au diplôme selon l'ordre et le cycle d'enseignement (en pourcentage)

\begin{tabular}{|c|c|c|c|c|c|c|}
\hline & \multicolumn{6}{|c|}{ Année civile ${ }^{a}$} \\
\hline & 1988 & 1989 & 1990 & 1991 & 1992 & 1993 \\
\hline \multicolumn{7}{|l|}{ Secondaire ${ }^{a}$} \\
\hline Secteur des jeunes & 62,8 & 62,0 & 62,5 & 63,9 & 66,8 & 65,0 \\
\hline $\begin{array}{l}\text { Secteur jeunes et adultes } \\
\text { de moins de } 20 \text { ans }\end{array}$ & 63,7 & 63,2 & 63,9 & 65,8 & 69,1 & 67,1 \\
\hline $\begin{array}{l}\text { Total jeunes et adultes } \\
\text { (tous âges) }\end{array}$ & 71,9 & 71,2 & 72,0 & 74,7 & 79,8 & 80,1 \\
\hline Collégial (DEC) & 35,9 & 36,6 & 37,2 & 38,0 & 38,8 & 38,7 \\
\hline \multicolumn{7}{|l|}{ Université } \\
\hline Baccalauréat & 20,8 & 21,7 & 23,1 & 25,0 & 26,1 & 27,4 \\
\hline Maitrise & 3,9 & 4,0 & 4,3 & 4,5 & 5,0 & 5,3 \\
\hline Doctorat & 0,5 & 0,6 & 0,6 & 0,7 & 0,7 & 0,7 \\
\hline
\end{tabular}

Source : ministère de l'Éducation du Quểbec.

a. Au secondaire, il s'agit de l'année scolaire prenant fin pendant l'année civile considérée; la diplomation de 1988 est donc celle de 1987-1988. 


\title{
RÉSUMÉ - SUMMARY - RESUMEN
}

\author{
LESPÉRANCE Aindré - L'ESPÉRANCE DE SCOLARISATION IOU DE VIE \\ SCOLAIREJ DES JEUNES AU QUÉBEC
}

De l'école primaire à l'université, la scolarisation totale dispensée en 1992-1993, correspond en moyenne à 15,3 années par Québécois et Québécoise en âge de fréquenter un établissement scolaire. Cette mesure est la somme des taux de scolarisation par année d'âge pendant une année scolaire donnée. L'espérance de scolarisation au Québec a augmenté de 0,9 année depuis 1987-1988 et se situe parmi les plus élevées au monde. Les gains récents ont été effectués en partie par du rattrapage au primaire et au secondaire réalisé au secteur des adultes. Pour le reste, les gains se situent à l'enseignement collégial $(0,3$ année) et à l'enseignement universitaire (0,3 année). L'allongement de l'espérance de scolarisation aux niveaux post-secondaires s'explique essentiellement par une accessibilité plus large.

Les Québécoises affichent dans l'ensemble une scolarisation supérieure à celle des hommes. Au primaire et au secondaire, la durée de leur scolarisation est moindre que celle des hommes parce qu'elles perdent moins de temps en redoublements. Parce que les femmes accèdent en plus grand nombre au collège et à l'université, en 19921993, leur espérance de scolarisation aux niveaux supérieurs est de 3,7 années, contre 2,9 années pour les hommes.

\section{LESPÉRANCE André - THE EDUCATIONAL EXPECTANCY OF YOUNG PEOPLE IN QUEBEC}

From primary school to university, Quebecers of school age will, on average, spend a total of 15.3 years in school. This figure represents the sum of rates of schooling per year of age for a given school year. Educational expectancy in Quebec has increased 0.9 years since 19871988 , and is today one of the highest in the world. Recent gains are due in part to increased completion of primary and secondary programs by students in the adult education sector. The remaining gains are at the college and university levels ( 0.3 years each). The increased educational expectancy at the post-secondary level is basically due to greater accessibility.

Females in Quebec are, on the whole, better educated than males. They spend fewer years in primary and secondary school because they lose less time repeating years. Since more women are attending college and university, their educational expectancy in 1992-1993 stood at 3.7 years compared to 2.9 years for men.

LESPÉRANCE André - LA ESPERANZA DE ESCOLARIZACIÓN IO DE VIDA ESCOLARI DE LOS JÓVENES EN QUEBEC

De la escuela primaria a la universidad, la escolarización total impartida en 1992-1993 corresponde en promedio a 15.3 años por 
Quebequense en edad de asistir a la escuela. Esta medida representa la suma de las tasas de escolarización por año de edad durante un año escolar dado. La esperanza de escolarización en Quebec ha aumentado de 0.9 año desde 1987-1988, y se encuentra entre las más elevadas del mundo. El aumento del rendimiento ha sido posible en parte gracias a las clases de recuperación para adultos en las escuelas primaria y secundaria. Por lo demás, se ha ganado 0.3 año en el sector de la preparatoria y 0.3 año en el sector universitario. El incremento de la esperanza de escolarización en los niveles post-secundario se explica principalmente por una mayor accesibilidad.

Las mujeres en Quebec muestran en general un grado de escolarización superior al de los hombre. En la primaria y la secundaria, su periodo de escolarización es más corto que el de los hombres, pues pierden menos tiempo en repetir cursos. Puesto que las mujeres acceden en mayor número a la escuela preparatoria y a la universidad, su esperanza de escolarización en los niveles superiores es, en 1992-1993, de 3.7 años contra 2.9 años para los hombres. 